\title{
Procesos emocionales de cuidado y riesgo en profesionales que trabajan con el sufrimiento humano.
}

\section{Health care of emotional processes and risk in professionals working with human suffering}

\author{
Germán Morales F. ${ }^{1}$ \\ Janet Carola Pérez E. ${ }^{2}$ \\ María Alejandra Menares N. ${ }^{3}$
}

\begin{abstract}
Resumen
Desde los 60' hasta hoy en día ha sido un tema de interés progresivo, el proceso de desgaste emocional que afecta a los profesionales que trabajan con el sufrimiento humano. El presente trabajo tiene por objetivo investigar dicho proceso en tres instituciones cuyo trabajo está relacionado con el sufrimiento físico y daño social de niños y jóvenes. El diseño metodológico de este estudio se desarrolló de acuerdo a la perspectiva cualitativa de la grounded theory de Glasser y Strauss. Se realizaron ocho entrevistas en profundidad a profesionales que trabajan en las áreas señaladas. Los resultados de la investigación arrojaron similitudes y diferencias en el discurso de los profesionales de las tres instituciones estudiadas, dando cuenta en su mayoría procesos de riesgo para la salud mental, y -se señala- a la conversación grupal como única estrategia que promueve el cuidado.
\end{abstract}

Palabras claves : autocuidado, desgaste emocional, profesionales.

\begin{abstract}
Abstratc
From the sixties onwards, there exists an increasing interest on the burn-out risk that professionals working with human suffering experience. This paper is about a research carried out in three institutions involved in healing social and physical damage in children and adolescents. A qualitative methodology was used, based on Glasser \& Strauss grounded theory. Eight professionals from the mentioned institutions were interviewed. It turned out that all i acknowledged being at mental health risk , displaying some differences in their experiences. Also, all mentioned that sharing experiences in a group conversation was their main strategy for mental self-care.
\end{abstract}

Keywords : selfcare, burnout, professionals.

\footnotetext{
${ }^{1}$ Psicólogo. Profesor, Dpto. de Psicología, Facultad de Ciencias Sociales, Universidad de Chile. Investigador y Terapeuta, Instituto Latinoamericano de Salud Mental y Derechos Humanos (ILAS).

${ }^{2}$ Psicóloga Clínica, Universidad de Concepción.

${ }^{3}$ Psicóloga Clínica, Universidad Diego Portales.
} 
En el trabajo con violencia y salud, es visible el desgaste emocional de los profesionales en su trabajo terapéutico y/o rehabilitador con el sufrimiento humano de personas y grupos. Este desgaste cotidiano se expresa en licencias médicas reiteradas, deserción de áreas de trabajo, y severas dificultades en torno a la eficacia de los distintos programas desarrolladas. Se ha dado cuenta de esta observación en algunas investigaciones que refieren ausentismo laboral, evitación del trabajo, baja productividad, incremento de consumo de tabaco y de otras sustancias, actitud de omnipotencia y cambio de relaciones entre los profesionales y sus seres significativos (Guerrero, 1999).

En el tradicional modelo de salud pública de factores de riesgo y protección, se indica que las medidas de higiene sanitaria han ido en progresiva mejoría, lo que ha implicado un aumento en los estándares de protección sanitaria y asignaciones de riesgo de los trabajadores. Dicha práctica se ha circunscrito a la protección respecto de la salud física, particularmente en el ámbito de procesos infecto/contagiosos y de utilización terapéutica de radioactividad.

En esta misma línea, en los últimos años ha existido una progresiva demanda por parte de los trabajadores de la salud para considerar también el riesgo y la protección para ellos, en el área de la salud mental, denominándola autocuidado (MINSAL, 2000). Ello ha sido relevante en profesionales y técnicos que trabajan en torno al sufrimiento humano.

\section{Marco Teórico}

Los conceptos más acudidos para designar, describir, explicar y eventualmente intervenir en dichos procesos son los Riesgos de Equipo, Burnout y Autocuidado.

El concepto de Riesgos de Equipo ha surgido de la práctica de supervisión clínica, y desde la perspectiva teórica psicoanalítica y sistémico contextual, intentando describir, interpretar y proponer modalidades de intervención que promuevan el Autocuidado y disminuyan los Riesgos de Equipo. Se definen Riesgos de Equipo a "aquellos elementos de la dinámica grupal que ponen en riesgo al equipo en el desarrollo de su tarea constituyente, que se expresan en ansiedades que emergen en el vínculo con los grupos de alto riesgo hacia los cuales se dirige la intervención" (Morales, 1996). Este concepto supone que los grupos se constituyen como tales en torno a una tarea, y desde allí desarrollan una mentalidad grupal (Bion, 1963) y/o sistema institucional (Corsi, 1999), desde donde emergen las ansiedades que tienen como contenido la tarea del grupo (Pichon-Rivière, 1985; Morales, 1997; Morales \& Lira, 1997; 2000). Dado que los grupos tienden a protegerse y/o defenderse de dichas ansiedades, estas generan defensas y dinámicas disfuncionales que constituyen un riesgo para las personas y grupos, particularmente para quienes desarrollan su trabajo con situaciones de violencia (Morales \& Lira, 1997).

El concepto de Burnout ha surgido, desde la investigación empírica clínica y de la psicología social, y fundamentalmente desde la perspectiva teórica cognitivo/ conductual (Freudenberger, 1974); intentando describir, interpretar, y proponer modalidades de intervención que promuevan la competencia para los procesos de afrontamiento del estrés y disminuyan el Burnout (Maslach, 1993). El Síndrome Burnout se define como "una respuesta a un estrés emocional crónico cuyos rasgos principales son el agotamiento físico y psicológico, una actitud fría y despersonalizada en la relación con los demás y un sentimiento de inadecuación en las tareas que se ha de realizar" (Maslach \& Jackson, 1981).

La conceptualización del término ha sido descrita como una labor difícil y problemática, debido a la diversidad de criterios y a la utilización extensa de éste a través de la 
bibliografía. No obstante, se han identificado los elementos comunes y esenciales del concepto. A saber, es aplicable sólo a trabajadores de servicios de ayuda, es decir, a aquellos que se utilizan a sí mismos como herramienta en el trabajo con otros (Farber, 1991). Asimismo, es un elemento común la sensación subjetiva de ausencia de apoyo y feedback en sus esfuerzos por ayudar a otros (Farber, 1991). Por último, se asocia con la sobreimplicación por parte del profesional, lo que aumenta las posibilidades del sentido de fracaso, deterioro de la salud mental y deseo de abandono del trabajo, entre otras (Koeske \& Kelly, 1995).

El síndrome del burnout descriptivamente involucra el compromiso de al menos tres áreas de funcionamiento: somático, afectivo y conductual. Dentro de los síntomas emocionales se describen sentimientos de depresión, distanciamiento afectivo de las personas que se atiende, irritabilidad, disminución de la autoestima, baja satisfacción laboral y deseos de abandonar el trabajo (Guerrero, 1999).

Desde que el concepto fue acuñado por primera vez hasta hoy, se ha acumulado una extensa lista de investigaciones sobre el fenómeno enfatizando los distintos aspectos antes señalados. Entre otras investigaciones se destacan, aquellas realizadas con profesores (Ponce, 2001), estudiantes (Schaufeli et al. 2002), médicos (McManus et al, 2002), enfermeras (Maslach, 2002; Rozas, 2000), clérigos (Grosch \& Olsen, 2000), asistentes sociales, psiquiatras y psicólogos (Azar, 2000). Para el caso de profesionales de la salud mental, estos aumentan su vulnerabilidad y riesgo debido a la exposición constante al trauma de sus pacientes (Miller, 1998).

En la actualidad, algunos autores como Groch y Olsen (2000), proponen que el Burnout debe ser entendido desde la interacción entre el sistema del self y el sistema institucional en el que se desenvuelve la persona. Asimismo, se ha cuestionado el foco principalmente cognitivo y de solución de problemas como estrategia de solución al Burnout, ya que se han identificado respuestas emocionales disfuncionales aparejadas a éstas (Kelly \& Perrewé, 2001).

El concepto de autocuidado tiene su origen en la salud pública y -más específicamente- desde la práctica de la enfermería. Según Orem (citado en González \& Arriagada, 1999) autocuidado se define como «aquellas actividades que realizan los individuos, las familias, o las comunidades, con el propósito de promover la salud, prevenir la enfermedad, limitarla cuando existe o restablecerla cuando sea necesario». La misma autora define tres niveles de necesidades (Universales, de Desarrollo y Desviaciones de la Salud) frente a las cuales surgen las prácticas y significados asociados al autocuidado.

Existen distintos tipos de unidades de significación de autocuidado. Según González \& Arriagada (1999) podrían considerarse las siguientes unidades de significado: Medidas y prácticas de autocuidado; conocimientos; obstaculizadores y facilitadores del cuidado.

Si bien se han identificado distintos conceptos y teorizaciones en relación a los riesgos de equipo, el síndrome de burnout y autocuidado, todas estas perspectivas destacan la necesidad de desarrollar intervenciones orientadas a promover la salud de las personas que trabajan con personas y su sufrimiento, velando por la eficacia de la tarea que desarrollan.

El objetivo general de la presente investigación es identificar y describir, desde la perspectiva de los trabajadores de la salud, ayuda y/o apoyo social, los procesos emocionales de riesgo y cuidado que experimentan en relación a su tarea. Los objetivos específicos son: 1) identificar y describir, desde la perspectiva de los profesionales, los procesos emocionales de riesgo relacionados con su labor asistencial. 2) identificar y describir, desde la perspectiva de los profesio- 
nales, los procesos emocionales de cuidado relacionados con su labor asistencial

\section{Metodología}

\section{Diseño}

El desarrollo de la investigación utilizó un diseño descriptivo-analítico, según los parámetros de metodología cualitativa. El diseño cualitativo utilizado consideró tres fuentes de información provenientes de tres instituciones que desarrollan su acción en el área de salud y riesgo social. En dichas instituciones se entrevistó a profesionales y se analizó el discurso producido por los mismos, buscando similitudes y diferencias respecto de los procesos emocionales de riesgo y cuidado, utilizando como método de análisis la Grounded Theory.

\section{Muestra}

La muestra total estuvo constituida por un total de ocho personas, tres profesionales pertenecientes a la Unidad de Oncología Infantil de un Hospital de la Región Metropolitana, dos de la Unidad de Tratamientos Intensivos, de otro Hospital de la misma región en Chile y tres del área Infracción a la Ley Penal Juvenil, del Servicio Nacional de Menores del mismo país. Por razones éticas se reserva el nombre de las instituciones específicas en las cuales se desarrolló el proceso de investigación, para el resguardo de la privacidad de los profesionales entrevistados, lo que constituyó un compromiso de anonimato con cada uno de ellos.

La muestra -de tipo intencionada- fue elegida en base a seleccionar casos típicos, dentro de un nivel alto de intensidad respecto de tareas ligadas al sufrimiento humano. Los criterios de inclusión para conformar la muestra fueron: (1) contar con formación profesional universitaria, (2) haber desarrollado su trabajo en la misma Institución por - al menos- un período de 3 años, y (3) participar voluntariamente. Ser un profesional que no trabaje directamente con personas, se constituyó en el criterio de exclusión.

La muestra quedó constituida por tres pediatras de oncología; un pediatra y una enfermera de UCI infantil, de dos hospitales de la región metropolitana; un psicólogo, una asistente social y un educador del Servicio Nacional de Menores.

\section{Técnicas de Recopilación de Información}

Se desarrollaron ocho entrevistas en profundidad $^{1}$, ya que consideramos ésta como la técnica cualitativa más adecuada para explorar procesos emocionales. Estas fueron desarrolladas en los respectivos lugares de trabajo. Siete de las entrevistas fueron grabadas en cassette y una sola fue registrada "a papel"; de éstas -seis- son transcripciones textuales y dos de ellas son resúmenes.

\section{Procedimientos}

Una vez establecidos los contactos en las tres instituciones, se realizaron las entrevistas a los 8 profesionales seleccionados. El análisis de los datos fue desarrollado en forma conjunta por los investigadores, basados en la Grounded Theory. Este es un modelo de generación del conocimiento de carácter inductivo, desarrollado por Glasser \& Strauss(1967), que supone una producción de información a partir de técnicas fundamentalmente -más no exclusivamente- cualitativas. Este modelo se fundamenta en la rigurosidad y fidelidad de los procedimientos de recolección de datos y -de su- codificación de acuerdo a categorías lingüísticas

\footnotetext{
1 Es una entrevista con cualquier entrevistado, a quien de acuerdo con los propósitos del investigador, se le da un tratamiento especial, no estandarizado, esto quiere decir, que se enfatiza la definición de la situación por el entrevistado, que se anima al entrevistado a estructurar el relato de la situación y se facilita que el entrevistado introduzca sus nociones de lo que considera relevante, en lugar de depender de las nociones del investigador sobre relevancia" (Valles, 1992, p. 188).
} 
que emerjan de los mismos datos, utilizando tres tipos de codificación (abierta, axial y selectiva), cada una de las cuales implica un mayor nivel de abstracción en relación a los datos; para concluir con un modelo hipotético en relación al o a los fenómenos estudiados. Posterior a este ejercicio inductivo, el modelo hipotético construido se relaciona con la producción teórica existente respecto del mismo fenómeno.

Se inició el análisis estableciendo diversos sistemas de codificación abierta, los cuales fueron contrastados respecto de su aplicabilidad a los distintos contextos laborales; una vez establecido el sistema de códigos abierto ${ }^{2}$ se realizó triangulación ${ }^{3}$ respecto de ésta codificación. Paralelamente, se establecieron tanto los sistemas de codificación axial ${ }^{4}$ y selectiva ${ }^{5}$, que sintetizan los datos del estudio.

En relación con los aspectos éticos establecidos a priori, se concretó el carácter voluntario de los participantes, el anonimato de los entrevistados, la confidencialidad del contenido de las entrevistas y del contexto institucional. Posteriormente, en función de los acuerdos establecidos se entregó un informe ejecutivo de los resultados del estudio a las instituciones, con copia a cada uno de los participantes.

\section{Resultados}

Del análisis de los datos se pueden identificar tres grandes categorías, a saber:

1. Naturaleza de la Tarea: que abarca todas las acciones que desarrollan los profesionales, en forma individual o conformando equipos de trabajo, en función de los objetivos y características del contexto de cada institución, que tienden a «mejorar las condiciones de salud (física y social) de los usuarios» (ver cuadro $\mathrm{n}^{\mathrm{o}} 1$ ).

La «Naturaleza de la Tarea», que comprende las acciones y la forma en que el profesional desarrolla cotidianamente para el ejercicio de su rol. Generalmente, se ve afectada por dos elementos contextuales: 1) los horarios, pues existen tareas se desarrollan a través de sistema de turnos, 2) la carga laboral, entendida tanto respecto de la cantidad de casos a atender como de los plazos a cumplir.

Estas acciones desarrolladas por los profesionales, se enmarcan en un contexto Institucional específico, que abarca un grupo determinado de "compañeros de trabajo» como «la disposición a un determinado set de recursos». Respecto de este último, las tres instituciones estudiadas presentan un «conjunto limitado de recursos económicos», lo que afecta al cumplimiento eficiente de la

\footnotetext{
2 Codificación abierta, es una estrategia cualitativa de categorización de datos, que consiste en separar los datos en partes o elementos discretos, al establecer similitudes y diferencias ente ellos. La codificación abierta conduce a establecer primero conceptos y luego categorías, que abarcan -en un nivel de abstracción mayor- los conceptos previamente identificados (Glasser \& Strauss, 1967).

3 La triangulación, es una estrategia basada en la comparación de métodos, fuentes y/o investigadores, que permite reducir el impacto de éstos en los resultados de la investigación (Marshall, 1990).

4 Codificación axial, es un nivel de análisis cualitativo de los datos, que corresponde a un segundo nivel-luego de realizada la codificación abierta-, que consiste reordenar de una nueva manera los datos, estableciendo relaciones entre las categorías. Las relaciones se establecen en sistema de paradigma, que considera un fenómeno central, sus condiciones antecedentes y contexto, condiciones intervinientes, las estrategias de acción e interacción y las consecuencias (Glasser \& Strauss, 1967).

5 La codificación selectiva, es un nivel análisis -cuyo nivel de abstracción es superior a la codificación axial-, y consiste en seleccionar la categoría central del estudio y relacionarla sistemáticamente con las otras categorías descubiertas, validando dichas relaciones y completando aquellas categorías que requieren un mayor grado desarrollo (Glasser E Strauss, 1967).
} 
tarea, así como la satisfacción de los profesionales que laboran en éstos, al no poder contar con espacios físicos ordenados, limpios, estéticos y privados.

Las acciones que tanto los profesionales del área médica y social realizan, se dirigen principalmente, a la persona usuaria del servicio correspondiente como a su familia. Estos usuarios, presentan características especiales en -lo social o físico-, que son las que determinan su incorporación. Los que ingresan al SENAME corresponden generalmente a personas en «riesgo social», que presentan conductas inadecuadas e infringen la ley. Los usuarios de los servicios de salud, se caracterizan por un compromiso severo de su salud física y con un pronóstico reservado; sólo aquellos usuarios que se atienden en modalidad ambulatoria en éstos, presentan una salud más estable, cuyo pronóstico es más favorable. Más allá, de lo arriba especificado, tanto los usuarios como sus familias, presentan características personales que afectan en la ejecución de la tarea, como en el compromiso emocional de los profesionales que prestan dichos servicios (ejemplo: "el otro día llegó una niñita exquisita, que era preciosa y como muy parecida a una niña que yo quise mucho, mucho, que falleció hace un año... y yo me di cuenta que ella enganchó conmigo y yo con ella", entrevista 1, párrafo 221).

\section{Cuadro $\mathbf{n}^{\circ} \mathbf{1}$ : NATURALEZA DE LA TAREA}

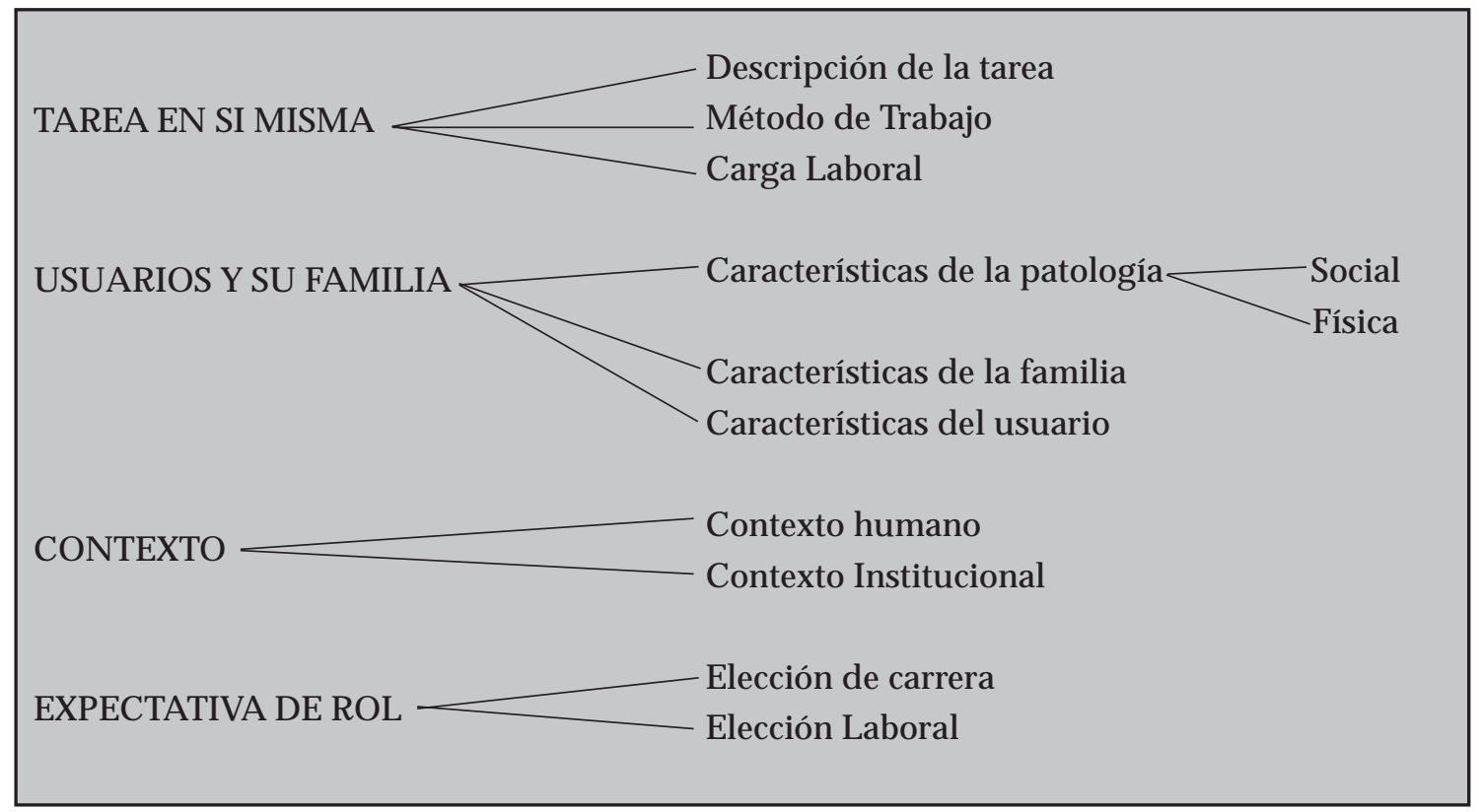

2. Sensaciones, Emociones y Cogniciones del Profesional: corresponde al conjunto de sensaciones, emociones vivenciados y a las cogniciones construidas por los profesionales y evaluados por estos en polos opuestos, así como la evolución de éstos (ver cuadro $\mathrm{n}^{\circ} 2$ ). Las sensaciones y emociones surgen respecto de los diferentes elementos que conforman la «naturaleza de la tarea».

\section{Las Sensaciones identificadas son:}

1. Activación/cansancio físico respecto de las tareas realizadas;

2. Exito/fracaso respecto a las tareas realizadas;

3. Motivación/desmotivación para realizar las tareas asignadas y de la elección 
laboral; y

4. Presencia/ausencia de afecciones somáticas.

\section{Las emociones identificadas son:}

1. Capacidad de involucración afectiva; es decir, mantener el establecimiento de relaciones afectivas hacia los usuarios y sus familias v/s Aplanamiento afectivo, en el cual esto se ha perdido.

2. Las emociones experimentadas por los profesionales, pueden ser clasificadas en: la gama placentera, que incluye, Alegría, Esperanza, Triunfo. Y la gama displacentera, que está conformada por: Tristeza-Pena; Rabia-Ira; AngustiaAnsiedad; Desesperanza; Miedo; Dolor; Culpa y Frustración.

\section{Las cogniciones identificadas son:}

1. Apto/no apto del setting laboral;

2. Adecuación/inadecuación ante la elección de carrera y laboral;
3. Aceptación/rechazo/indiferencia de los compañeros de trabajo respecto de las reacciones emocionales personales suscitadas por en el ejercicio de la labor; y

4. Las evaluaciones realizadas al tipo de relaciones interpersonales del «equipo de trabajo», descritas como conflictivas, cooperadoras o indiferentes.

Respecto de las tareas, existen tareas individuales y grupales, siendo -en este último caso- relevante el grado de confianza hacia ellos para el desarrollo de la tarea. Existen tareas pautadas y otras «realizadas por intuición». La tarea conlleva sensaciones de éxito, «darme cuenta que toda esa paciencia dio fruto, y que él está super bien" (entrevista 6, párrafo 40) y de fracaso «que es lindo el trabajo que pretendo desarrollar... porque no se puede hacer» (entrevista 4, párrafo 43); de motivación y desmotivación -por ejemplo- «llego bastante desmotivado» (entrevista 4, párrafo 2). Destacando -sí- en todos los contextos de trabajo la importancia de la tarea realizada, dada las consecuencias de ésta.

\section{Cuadro $n^{\circ} 2$ : SENSACIONES, EMOCIONES Y COGNICIONES DEL PROFESIONAL}

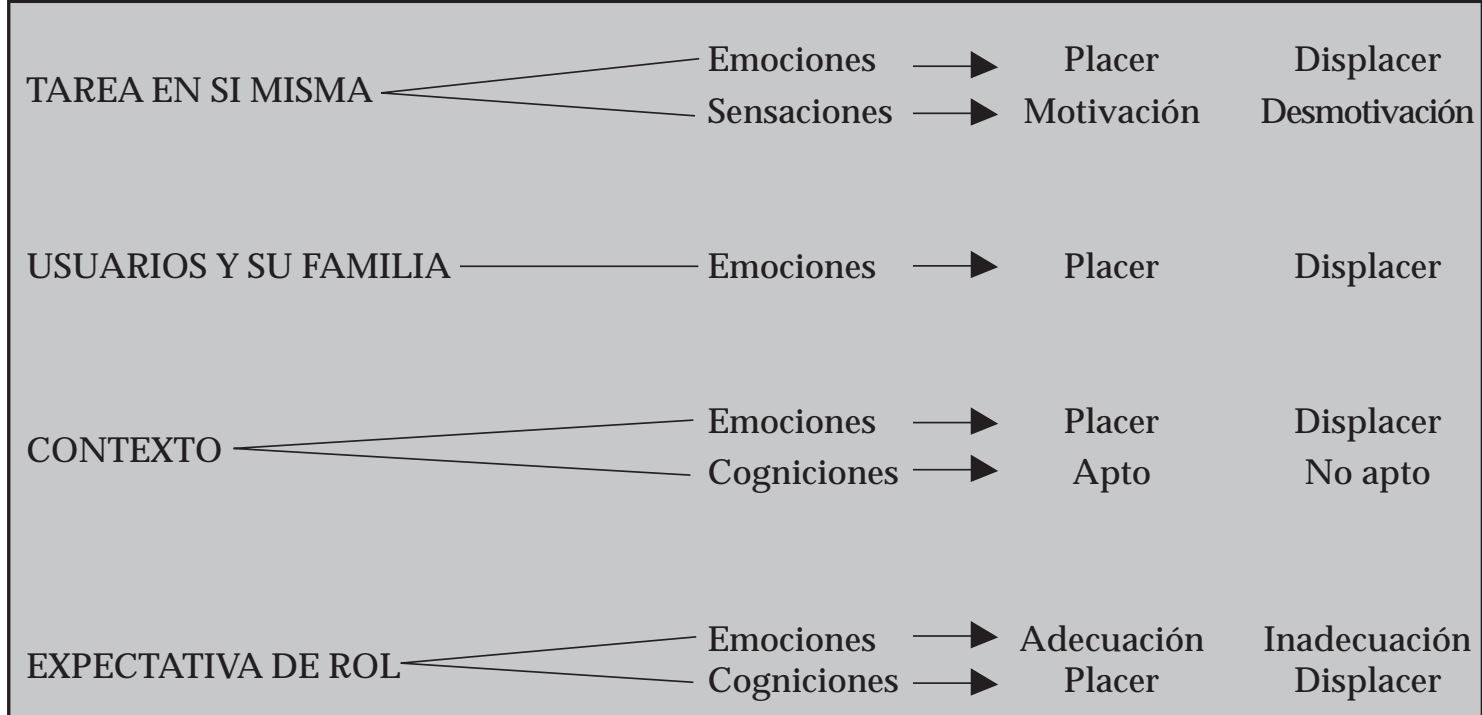


Las emociones vivenciadas -tanto respecto de la tarea como de los usuarios y sus familias- se corresponden a la gama placentera, particularmente cuando hay éxito en la tarea, y la gama displacentera, cuando la gravedad (dado la patología física o social) hace visualizar un mal pronóstico, que -en algunos casos- se corresponde con la muerte. Por su parte, la expresión emocional -de los profesionales- respecto de sus situaciones laborales, es aceptada por algunos de los miembros del equipo de trabajo; en cambio, otros la rechazan o son indiferentes a ésta. Sin embargo, se está produciendo un proceso de cambio al respecto, hacia una mayor aceptación de dicha expresión emocional.

\section{Procesos Emocionales de Riesgo y} Cuidado: Dice relación con los procesos vivenciados por los profesionales, ya sea voluntaria o involuntariamente, para abordar el conjunto de sensaciones, emociones y cogniciones que experimentan diariamente o producto de su experiencia laboral (ver cuadro $n^{\circ} 3$ ). Los procesos identificados en el conjunto de los entrevistados son:

\subsection{Regulación-distancia: consiste en} establecer distancia de aquellas personas / situaciones que gatillan sensaciones y emociones displacenteras. Por ejemplo: «yo me di cuenta que ella enganchó conmigo y yo con ella, y la derivé, traté de no ser su médico tratante" (entrevista 1, párrafo 211).

3.2 Desconexión-represión: consiste en sacar del campo de la consciencia las emociones/sensaciones que las personas/situaciones gatillan. Ejemplo: «aunque durante este tiempo he aprendido a separar las cosas que tienen que ver con la casa y las cosas que tienen que ver con el trabajo» (entrevista 4, párrafo 2).

3.3 Sobre-involucramiento: consiste en establecer un compromiso emocional marcado respecto de las personas/situaciones que generan las diversas emociones y sen- saciones. Por ejemplo: “... un gran quemado... uno se va a involucrar más con ese paciente de larga estadía... porque éste tiene más sufrimiento" (entrevista 8, párrafo 94).

3.4 Desborde: Consiste en expresar sin control las emociones vivenciadas en relación a la tarea. Ejemplos: "con los colegas uno hace catarsis de grupo" (entrevista 8, párrafo 56), "frecuentemente he llorado con los papás" (entrevista 1, párrafo 205).

3.5 Realizar acciones: Los profesionales realizan acciones concretas, ya sea relacionadas directamente con la tarea institucional y sus receptores, $\mathrm{o}$ actividades no relacionadas a éstas. Estas acciones están destinadas a reducir el conjunto de sensaciones y emociones vivenciadas.

3.6 Intelectualización: corresponde a buscar «explicaciones» respecto de la naturaleza de la tarea y las características de la patología (social/física), que permitan reducir la carga de emociones y sensaciones. Por ejemplo: al conversar con un chico que actúa agresivo, "trato de entender la reacción de él" (entrevista 5, párrafo 14).

3.7 Ensimismamiento: Se refiere a una ida de la conciencia y las emociones hacia uno mismo de manera de reducir la exteriorización de las emociones negativas.

Los profesionales -ante la carga emocional- que sus trabajos involucran desarrollan los procesos arriba indicados. En algunos casos, éstos se producen involuntariamente; en otros, existe una intensión consciente del sujeto para conseguir la desconexión emocional, en momentos de crisis y poder, así, realizar eficientemente la tarea. Por ejemplo, en Unidad de Cuidados Intensivos (UCI) "cuando llega un paciente uno tiende a bloquear sus emociones... uno se dedica a atenderlos y hacer todo lo que indica para tratar de sacarlos de su gravedad" (entrevista 8, párrafo 50). 


\section{Cuadro $\mathrm{n}^{\circ} 3$ : PROCESOS EMOCIONALES}

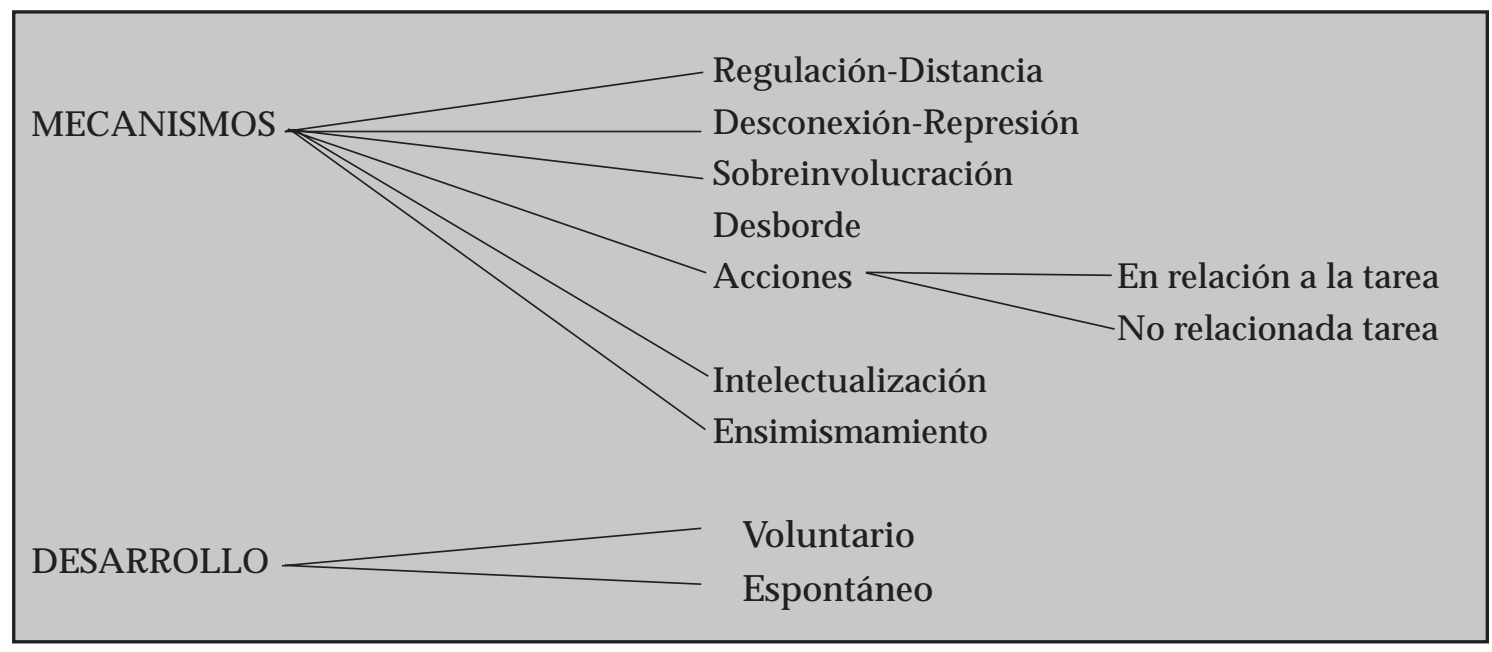

Las categorías y conceptos identificados, al ser analizadas bajo el modelo hipotético (de mayor grado de abstracción), se integran de la siguiente manera.

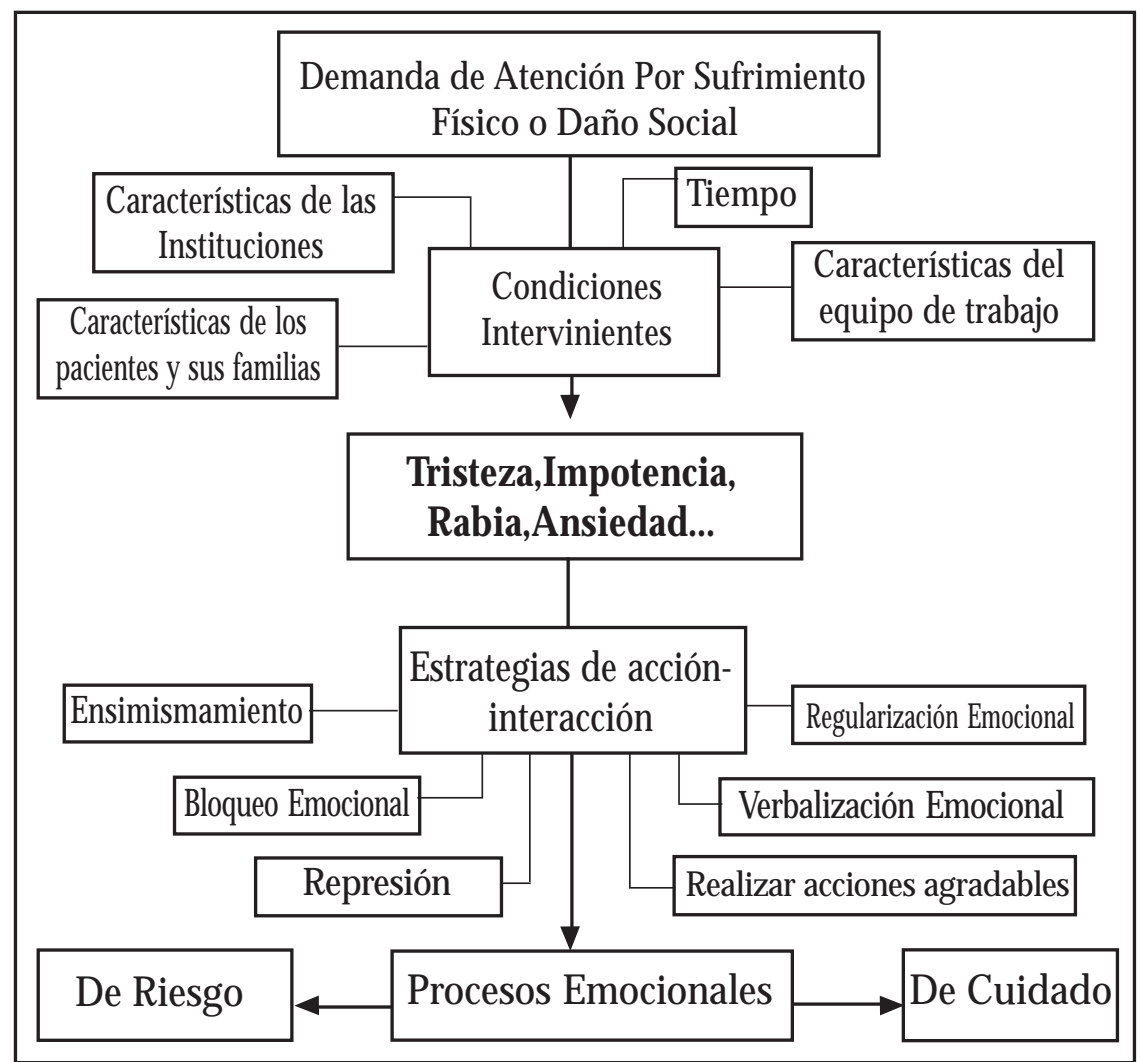


Las acciones de riesgo y cuidado relacionadas directamente con la tarea están orientadas al enfrentamiento de las situaciones gatillantes de las emociones displacenteras; «conversar» con compañeros al respecto del desarrollo de éstas, para con ello reducir la tensión emocional y/o elaborar el impacto de ésta. Las acciones no relacionadas con la tarea, son desarrolladas preferentemente como estrategias de cuidado, entre ellas se encuentran la lectura, practicar relajación, realizar paseos, celebrar cumpleaños, entre otras.

Condiciones Causales: Dentro de los eventos que llevan a la ocurrencia del fenómeno en estudio, es decir, de los procesos emocionales de cuidado y riesgo de los profesionales que trabajan en labores de ayuda, apoyo y/o tratamiento, es posible destacar a la demanda de atención como principal hecho desencadenante de tales procesos emocionales.

Contexto: Los procesos emocionales predominantes experimentados por los profesionales están referidos a las emociones displacenteras por sobre las placenteras. La angustia propia de la labor de los profesionales, los activa a la acción. Asimismo, el sufrimiento de los usuarios y sus familias generan en los profesionales procesos emocionales diversos, por lo general, orientados al abordaje de la experiencia de sufrimiento.

Inicialmente, se tiene la esperanza de poder ayudar efectivamente al usuario. Sin embargo, el profesional del SENAME siente que su contribución es muy menor en comparación a la problemática social del niño o adolescente, por lo que se presenta con un importante nivel de desesperanza ante sus funciones. No obstante, el asumir la responsabilidad de la "cura" o de "salvar la vida" de los niños y adolescentes en la UTI o en la Unidad de Oncología provoca un importante nivel de ansiedad que moviliza a los profesionales a buscar herramientas en sus conocimientos y destrezas.
Las emociones más comunes en los contextos médicos son tristeza, rabia, ansiedad, frustración y culpa. El éxito y la alegría que este conlleva se ven interferido por la ambivalencia cuando la "cura" ha tenido secuelas importantes en el proyecto de vida de los niños y adolescentes. Por otro lado, las emociones comunes en el contexto del SENAME son la frustración, la ansiedad y angustia, y la pena. La rabia aparece generalmente asociada hacia las estructuras sociales e institucionales. La gama placentera está poco presente, identificándose sensaciones de bienestar más que alegría.

Es posible observar que los profesionales reconocen que detenerse demasiado a pensar en el sufrimiento del paciente o de sus familias puede obstaculizar el trabajo. No obstante, otros entrevistados sugieren que esto le facilita sus labores y un mayor contacto con ésta.

Aparentemente, la expresión de las emociones por parte de los profesionales no es validada de la misma manera entre las distintas generaciones de médicos, siendo las últimas generaciones más abiertas a la expresión de las emociones y a reconocer que no siempre saben todas las respuestas.

Estrategias de acción/interacción: Las estrategias que se manejan para responder al fenómeno (proceso emocionales de riesgo y cuidado) se encuentran en dos niveles, uno que tiene que ver con procesos personales, intrapsíquicos y un nivel más conductual, que se relaciona con procesos pro-sociales.

Dentro de los procesos individuales se encuentran: a) regulación de la distancia con el usuario y su familia, b) la represión o desconexión de la emoción, c) una sobreinvolucración con la tarea o el usuario, d) el desborde emocional, e) la intelectualización y f) el ensimismamiento.

En tanto, a nivel conductual es posible observar actividades pro-sociales con rela- 
ción a la tarea o que no tiene relación con la tarea. Estas se refieren por lo general a la conversación con pares dentro o fuera del contexto laboral o diálogos con familiares. Estas conversaciones cumplen la principal función de descarga de la emoción y secundariamente la elaboración de la experiencia emocional. Lo común es que sólo se acompañen mutuamente en una descarga colectiva de emociones displacenteras, generalmente, pena, frustración y dolor. Fuera del contexto laboral, alguno de los entrevistados identifica a las actividades de relajación como una estrategia útil para aliviar este tipo de emociones.

Condiciones intervinientes: Dentro de las condiciones estructurales que facilitan o restringen las estrategias de acción/interacción se han identificado:

\section{Las características de los usuarios} y sus familias. Los procesos emocionales que se pone en juego en el desarrollo de la tarea de los profesionales entrevistados varía de acuerdo a ciertas características específicas de los usuarios y sus familias, por ejemplo, el tipo de patología va a interferir en el grado de cercanía o involucramiento del profesional con el paciente: "A algunas personas ese paciente gran quemado puede significar involucrase menos que con otro porque este tiene más sufrimiento... Hay personas que no les gusta tener grandes quemados dentro de la unidad."(entrevista 8, párrafo 4).

2. Las características del grupo de trabajo. Aquí influye si la tarea es desarrollada de manera individual o en equipo. Ya que cuando la tarea es en equipo y las relaciones interpersonales no pueden soslayarse el conflicto es una condición que interviene negativamente: "A veces hay problemas entre el personal y eso afecta porque están como medias tirantes las relaciones... aquí es una sala donde hay distintos oficinas, entonces, las personas no trabajan individual... no es que yo llegue y me encierre y trabajo sola y no me relaciono con nadie más" (entrevista 8, párrafo 22).

\section{Las características de la institución} y de la organización de la tarea. Cuando las instituciones cuentan con escasez de recursos físicos y/o técnicos y es necesario realizar derivaciones o peticiones de exámenes a otros servicios el desarrollo de la tarea se obstaculiza: “...se solicitan muchos exámenes hacia fuera y eso tiene toda una burocracia de tipo administrativo... Si el niño necesita que le controlen, que esta en ventilador, los gases. Esos gases tienen que bajar y en quince minutos estar de vuelta con él, resultado. Estamos acostumbrados a una rutina rápida y cuando no es así, sientes que hay un escollo..." (entrevista 8, párrafo 8).

4. Las características personales en relación a la elección del tipo de trabajo. Características personales intervienen en los procesos emocionales de los profesionales, especialmente aquellas relacionadas con la elección vocacional y / o laboral. Por ejemplo, los profesionales de la UCI sienten que deben ser capaces de tolerar el sufrimiento para poder trabajar en lo que de desempeñan: “...es que uno tiene que aprender a tolerar el dolor el sufrimiento. Si no lo toleras tú no puedes aguantar el trabajar. Ahí personas que vienen y están un día y se mandan cambiar porque no lo toleran, el trabajo con niños, el de cuidados intensivos..." (entrevista 8, párrafo 39).

5. El tiempo. El tiempo interfiere en los procesos emocionales de los profesionales entrevistados de dos formas uno en relación a los plazos por cumplir y en cuanto al tiempo que se pasa en contacto directo con el usuario. Cuando hay plazos por cumplir la ansiedad surge y dificulta el contacto con el usuario. Por otro lado, los pacientes de larga estadía en las unidades de oncología o de la UCI tienen más posibilidades de que el profesional se involucre afectivamente con ellos: “...No es lo mismo un niño diabético descompensado que se recupera en 24,48 
horas, en el que uno no ve sufrimiento... a un gran quemado... uno se va a involucrar más con ese paciente de larga estadía que aquel que va a tener poco..." (entrevista 8 , párrafo 94)

Consecuencias: Las estrategias de acción/ interacción da como resultado nuevos procesos emocionales que pueden facilitar el trabajo, o bien obstaculizarlo. Por otro lado, pueden ser de riesgo psicológico para el pro- fesional o de cuidado para su salud mental. Sin embargo, un mismo proceso emocional que en un momento puede ser de cuidado puede convertirse de riesgo en otro momento. Por ejemplo, el bloqueo emocional puede facilitar el trabajo cuando se requieren maniobras finas de trabajo en intervención clínica, como en una UCI, pero resultar de riesgo para el mismo profesional si este bloqueo se rigidiza y se generaliza a otras áreas de funcionamiento.

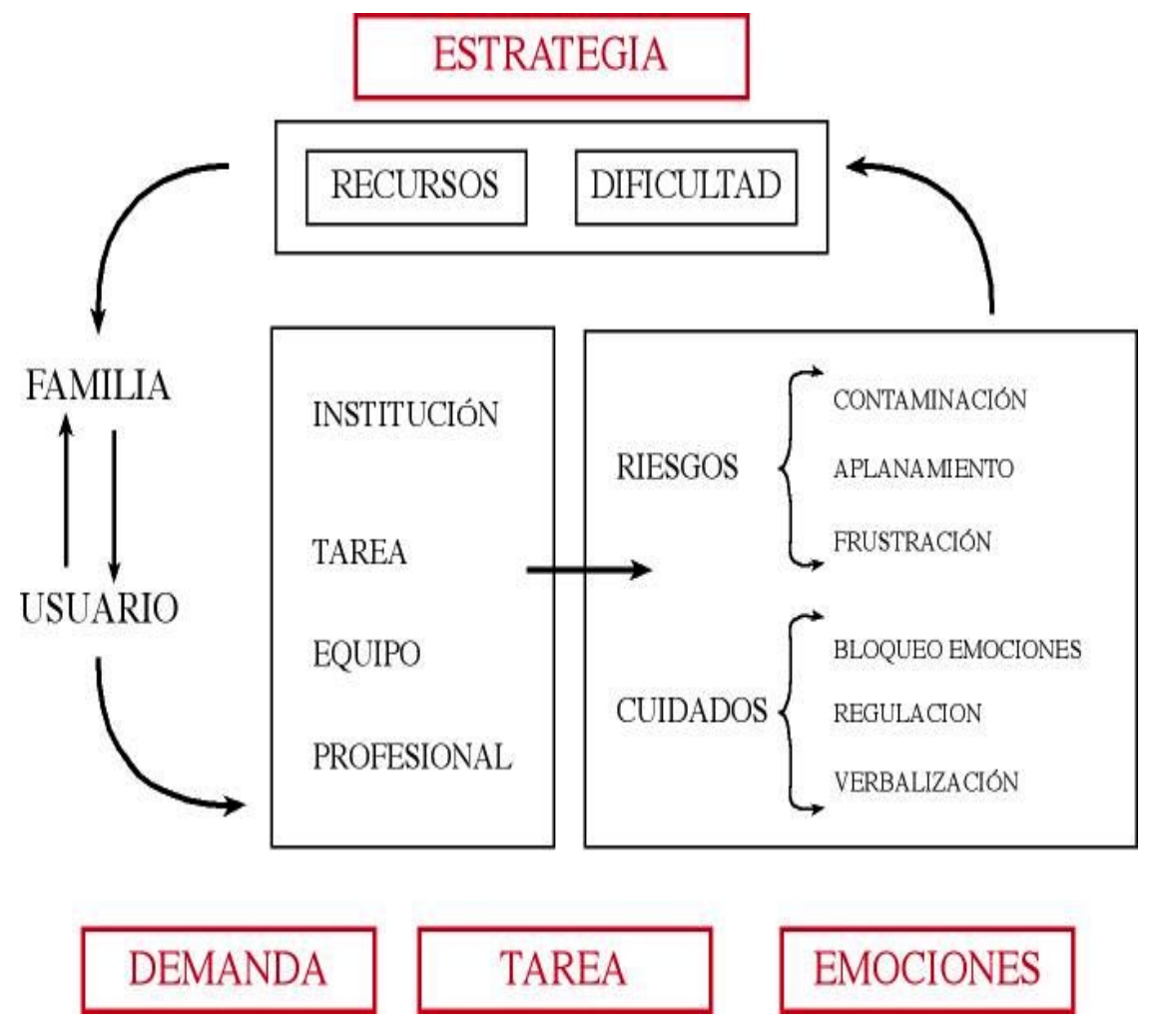

La codificación selectiva realizada tiene como objeto dar cuenta de un modelo comprensivo de los procesos emocionales de riesgo y de cuidado que experimentan los trabajadores de la salud, ayuda y/o apoyo social en relación a su tarea, y su efecto recursivo en esta misma. El modelo planteado es el siguiente:
La familia y/o los usuarios hacen demandas en torno a su sufrimiento desde donde se constituye una tarea.

La demanda surge desde el usuario hacia su familia, desde la condición de enfermedad infantil, o desde la condición de riesgo social juvenil.

En la condición de enfermedad, se trata de niños con cáncer ya diagnosticado y/o con una afección que demande atención de 
emergencia en la unidad de tratamiento intensivo (por lo general quemaduras severas). Aquí surgen la necesidad de -que la familia y un médico- desde un diagnóstico inicial, se movilizan para establecer un proceso diagnóstico más preciso y de tratamiento de la enfermedad que aqueja al niño.

En la condición de riesgo social, se trata de menores en conflicto con la justicia por un problema conductual ligado a la transgresión a la ley. Aquí surge la necesidad de que la familia y los tribunales desde una transgresión punible, se movilicen para establecer un proceso diagnóstico de discernimiento, y determinar un proceso jurídico.

La demanda desde el sufrimiento se constituye en una tarea, institucional, grupal e individual.

Esta demanda se vehiculiza a una institución, cuyo propósito es la salud y/o la protección de los menores. Esta institución define una tarea específica en función de la enfermedad o riesgo social, la que encomienda a un programa o unidad especializada. En ella un grupo y/o equipo busca acoger la demanda de los usuarios y sus familias mediatizadas por la institución. En dicho grupo laboran profesionales cuya función es la atención directa de lo niños y adolescentes que sufren a causa de su enfermedad o riesgo social.

Las tareas a desarrollar se relacionan con establecer diagnósticos y/o tratamientos para proteger los derechos del niño y mitigar el dolor, facilitar procesos terapéuticos y/o de rehabilitación según sea el caso. Esto implica que los profesionales establecen vínculos con los niños y/o adolescentes y sus familias en torno a la tarea de ayuda. En estos vínculos que se construyen de corto, mediano o largo plazo, los profesionales experimentan emociones.
La tarea de ayuda gatilla emociones asociadas al riesgo y al cuidado personal.

La tarea suscita emociones de riesgo y de cuidado, cuyo carácter se asigna en función de lo perturbador o lo aliviador que resulta para cada persona.

Los procesos emocionales asociados al riesgo, están aquellas que son percibidas como ligadas a la:

1. Contaminación emocional; por su capacidad de desborde emocional que son principalmente la angustia y la rabia;

2. Aplanamiento afectivo por su capacidad anestésica, que se constituye a partir de la cronificación de emociones displacenteras;

3. Frustración por su capacidad depresiva, que son principalmente la tristeza y la culpa.

En las emociones asociadas al cuidado, están aquellas que son percibidas como ligadas al:

1. Bloqueo y distanciamiento emocional, por su capacidad de neutralizar el desborde emocional frente a la angustia y la rabia;

2. Regulación emocional; por su capacidad de control frente a la antipatía, empatía y simpatía con los usuarios y sus familias;

3. Verbalización emocional; por su capacidad expresiva predominantemente frente sentimientos depresivos ligados al riesgo vital y a la muerte.

Los procesos emocionales asociados al vinculo con los usuarios generan estrategias interactivas que se traducen en dificultades $y$ recursos personales y grupales. 
En el proceso de demanda y servicios de ayuda, apoyo y rehabilitación entre profesionales y usuarios, se da un continuo devenir de estrategias que dan lugar a una interacción recurrente que establecen usuarioprofesional inserto en su contexto. Dado que el usuario experimenta una necesidad, que se traduce en una demanda, esta demanda se dirige hacia los profesionales quienes están constantemente respondiendo emocionalmente respecto de la tarea de ayuda encomendada. Estas respuestas emocionales se traducen en estrategias de carácter defensivo, que se expresan en la forma de interactuar con los usuarios y los pares, quienes a vez retroalimentan y perpetúan dicha forma de interacción defensivas. Estas estrategias están orientadas a protegerse del impacto emocional, pudiendo ubicarse en aquellas que activan recursos y las que generan dificultades dependiendo de las características de los profesionales y sus contextos institucionales.

\section{Discusión.}

\section{El vínculo en los contexto institucional promueve procesos emocionales específi- cos.}

La temporalidad que implican los procesos diagnósticos y de tratamiento adquieren un lugar central en las diferencias de los procesos emocionales experimentados por los profesionales de los distintos contextos analizados. La temporalidad de los procedimientos referidos se traduce en que la duración del vínculo de estos profesionales con los usuarios es diferente, y ello marca -de manera fundamental- el curso de los procesos emocionales.

En el caso del contexto institucional de salud (hospitales) se observan dos tipos de temporalidad: la crónica y la episódica. Aún existiendo esta diferencia de temporalidad en una enfermedad crónica o en una atención de emergencia, los profesionales presentarían una sensación subjetiva de control.
En ésta, el éxito o el fracaso en la tarea se tiende a atribuir a la propia acción de los profesionales de salud, dado que efectúan no sólo un diagnóstico sino que prescriben un tratamiento.

En el caso del contexto de Riesgo social (SENAME), si bien se observa una temporalidad episódica, los profesionales alcanzan a vincularse por un tiempo importante con los niños y jóvenes; pero la acción ejercida en la tarea (diagnóstico y derivación) no sería percibida como fundamental en el curso del proceso jurídico de los menores. En este contexto institucional, la atribución de éxito o fracaso en la tarea estaría externalizada. Existiría una sensación de escaso y/o débil rol, ya que ellos sólo efectúan un diagnóstico de discernimiento, pero no determinan el curso judicial de la causa en que están implicados los niños y adolescentes.

En la acción de ayuda ante el sufrimiento humano la emoción predominante sería la angustia.

La emoción central que gatilla el sufrimiento humano parece ser la angustia, según lo descrito por todos los entrevistados. Muchas veces, la angustia surgida en el proceso se transformaría en miedo o impotencia. El miedo estaría relacionado con la posibilidad de identificar el estímulo estresor, y si éste es percibido como ajeno por los profesionales promueve el aislamiento y sentimientos depresivos. Por su parte, la impotencia estaría relacionada con la posibilidad de experimentar tristeza ante el sufrimiento del otro, o rabia por la urgencia de mitigar dicho sufrimiento, y aquí los profesionales podrían aislarse o promover la pertenencia grupal dependiendo del contexto.

A pesar del predominio de lo angustioso, la posibilidad de establecer una relación de ayuda implicaría gratificaciones ligadas no sólo al éxito de dicha tarea, sino también -y de manera fundamental- en la magnitud del esfuerzo emprendido. Este esfuerzo se 
vería recompensado por la valoración y reconocimiento social.

El contexto de salud presentaría mayores posibilidades de reconocimiento social, por el status de la tarea, -en cambio- los profesionales que trabajan con menores en riesgo social, serían objeto de descalificación o al menos- de un escaso reconocimiento social. Esto, además, promovería de manera diferencial la noción de control interno y externo respectivamente.

Considerando tanto las particularidades de los contextos institucionales como el curso de los procesos emocionales vividos por los profesionales implicados, se concluiría que en el contexto hospitalario, existe una predominancia de la rabia y angustia, que tenderían a activar a los profesionales hacia la acción grupal, pudiendo ésta acoger o generar conflictos interpersonales por las reacciones emocionales mutuas. En el contexto de riesgo social, existiría una predominancia de la angustia y sentimientos depresivos, los cuales inmovilizarían a los profesionales, y promoverían en ellos el ensimismamiento y la soledad, y el riesgo de embotamiento afectivo.

\section{Los procesos emocionales de cuidado serían más difusos y escasos.}

No se observa con claridad en los profesionales entrevistados la distinción de procesos emocionales de cuidado, denominados habitualmente de "autocuidado". Estos tienden a ser más difusos y escasos, o a no relacionarlos con su tarea profesional. Entre los procesos emocionales que se distinguen, los más nítidos serían la conversación, el reconocimiento de los propios límites en la acción profesional, y la necesidad de establecer límites entre los ámbitos laboral y familiar.

La conversación apelaría al valor de verbalizar las sensaciones, emociones y conflictos, pudiendo ésta tener un efecto catártico o elaborativo. Al mismo tiempo, para los entrevistados, adquiere un tremendo valor el compartir dudas e inseguridades respecto del diagnóstico y/o el proceso terapéutico a desarrollar con los usuarios.

El reconocimiento de los propios límites se expresaría como referencia ideal, al valorar las rotaciones en la tarea, y al buscar indicadores de desgaste emocional.

La necesidad de demarcar lo familiar y laboral constituiría una tarea en extremo difícil, sobre todo cuando se cumplen turnos. Esta demarcación se experimentaría como un aspecto a revisar constantemente en términos introspectivos.

Todos estos procesos ligados al cuidado requieren y parecieran potenciarse por lo grupal. Como resulta evidente, ellos se tienden a dar en el contexto hospitalario, ya que en el contexto de riesgo social serían más escasos y difusos.

\section{Validez de los conceptos teóricos de pro- cesos de desgaste emocional.}

La investigación evidenciaría la validez tanto de los conceptos sobre desgaste emocional utilizados anteriormente como del conocimiento general de éstos por parte de los profesionales.

En el caso del contexto de riesgo social se observarían las categorías más clásicas de "burnout" en los entrevistados. Podríamos dar cuenta de los síntomas propios de este síndrome como el agotamiento físico y psicológico, una actitud distante y aislada en la relación con los demás y un sentimiento de inadecuación en las tareas que se realizan.

En el caso del contexto de salud los síntomas de "burnout" serían menores, y más bien se evidenciarían los llamados "riesgo de equipo". Podríamos observar algunos mecanismos que tienden a protección y/o defensa de la angustia, que generan algunas dinámicas disfuncionales que constituyen un riesgo para las personas y grupos. 


\section{Nuevas preguntas, limitaciones $y$ sugerencias.}

Las preguntas más centrales y al mismo tiempo sugerencias de investigación futura serían el poder considerar como factores relevantes la distinción de lo generacional y del género. Lo generacional se refiere no sólo al interés de discriminar el rol que juega la edad, sino más bien la cultura generacional en la profesión, que en el contexto hospitalario se hace más nítida. Ello adquiría mayor relevancia tanto por los cambios en la formación de profesionales de la salud en la relación con los pacientes, como por las modificaciones que podría implicar la reforma procesal penal respecto de los profesionales del área social.

Es necesario profundizar y diferenciar en el rol que juega el factor género en los procesos emocionales de hombres y mujeres, que en el caso de esta investigación se insinúa como un aspecto a explorar.

\section{Referencias}

Azar, S. (2000). Preventing burnout in professionals and paraprofessionals who work with child abuse and neglect cases: A cognitive behavioral approach to supervision. Psychoterapy in Practice, 56(5), 643-663.

BION, W.R. (1963). Experiencias en grupos. Ed. Paidós: Buenos Aires.

CORSI, J. (1999). Un modelo integrativo para la comprensión de la violencia intrafamiliar. En G. Ferreira . Hombres violentos, mujeres maltratadas (pp. 32-50). Buenos Aires: Editorial Sudamericana.

FARBER, B. (1991). Crisis in education: Stress and Burnout in the American Theacher. JosseyBass Publishers. USA.

Freudenberger, H. J. (1974). Staff burnout. Journal of Social Issues, 30, 159-165.
Glasser, B.G. \& Strauss, A.L. (1967). The discovery of grounded theory. Chicago: Aldine Publishing Company. Cap. 1.

Grosch, W. \& Olsen, D. (2000). Cleregy Burnout: A Integrative Approach. Psychoterapy in Practice, 56(5), 619-632.

GuerRero, E. (1999). “Burnout" o desgaste y afrontamiento del estrés laboral en docentes universitarios. Premios nacionales de Investigación Educativa, 317-333. Ministerio de Educación y cultura, Madrid, España.

Kelly, Z. \& Perrewé, P. (2001). Affective Personality and the coping content of emotional social support: coping in organizations. Journal of Applied Psychology, 86(3), 459-467.

Koeske, G. \& Kelly, T. (1995). The impact of overinvolvement on Burnout and satisfaction. American Journal of Orthopychiatry. Vol.65 (1).

Maslach, C. \& JACKSON, S. (1981). Maslach Burnout Inventoy. California: Consulting Psychologist Press.

Maslach, C. (1993). Burnout: A multidimensional perspective. En W.B. Scvhaufeli, C. Maslach, \& T. Marek (Eds.), Professional burnout: Recent developments in theory and research (pp. 19-32). Washington, DC: Taylor \& Francis.

Maslach, A. (2002). A psychoanalytic existencial approach to burnout: Demonstrated in the cases of a nurse, a teacher, and a manager. Psychoterapy Theory Research Practice, Training, 39(1), 103-113.

McManus, I.C., Winder, B.C. \& Gordon, D. (2002). The causal links between stress and burnout in a longitudinal study of UK doctors. Lancet, 359 (9323), 2089-2090.

Miller, L. (1998). Our own medicine: Traumatized psychoterapists and the stresses of doing therapy. Psychoterapy, 35, 137-146. 
MINSAL (2000). Taller de salud mental y trabajo. Dpto. de Salud Ocupacional, División de salud Ambiental. Mimeografiado.

Morales, G. (1996). El equipo de intervención psicosocial como un grupo de alto riesgo. Salud y Cambio. Revista Chilena de Medicina Social, 22 (6), 38-45.

Morales, G. (1997). Subjetividad, psicología social y problemas sociales. Revista de Psicología/Universidad de Chile, 1(6), 72-79.

Morales, G. \& Lira, E. (1997). Dinámicas de riesgo y cuidado de equipos que trabajan con situaciones de violencia. En E. Lira (Ed.), Reparación, derechos humanos y salud mental (105-121). Santiago: CESOC.

MorAlEs, G. y LiRA, E. (2000). La «receta del autocuidado»: Los riesgos de equipo de programas que trabajan con situaciones de violencia». En Vilches, O. (Editora), Violencia en la cultura: Riesgos y estrategias de intervención (pp. 55-80). Santiago: Ediciones Sociedad Chilena de Psicología Clínica.

Pichon-Rivière, E. (1985). El proceso grupal: Del psicoanálisis a la psicología social. Buenos Aires: Nueva Visión.
Ponce, C. (2001). Burnout y estrategias de afrontamiento en profesores del segundo ciclo de enseñanza básica de los establecimientos municipales de la comuna de San Joaquin. Tesis para optar al grado de magister en psicología mención educacional. Pontificia Universidad Católica de Chile, Santiago.

Rozas, G. (2000). Estudio descriptivo de los factores protectores en relación al burnout, en personas que trabajan en unidades de oncología infantil de dos hospitales de la región metropolitana. Tesis para optar al grado de magister en psicología mención clínica. Pontificia Universidad Católica de Chile, Santiago.

Schaufeli, W.; Martinez, I., Marques, A., SALANOVA, M. \& BAKKER, A. (2002). Burnout and engagement in university students: A cross national study. Journal of Cross Cultural Psychology, 33(5), 464-481.

VAlles, M (1992). Técnicas cualitativas de Investigación Social. Reflexiones metodológicas y práctica grupal. Barcelona: Ed. Síntesis. 